\title{
EDITORIAL
}

\section{Still no certainty about the role of upfront bortezomib among patients with AL amyloidosis}

\section{Leukemia (2014) 28, 2273-2275; doi:10.1038/leu.2014.271}

In this issue of Leukemia, there are two important-albeit complicated-case-controlled series comparing bortezomibcontaining therapeutic regimens to those without for the treatment of newly diagnosed $A L$ amyloidosis. ${ }^{1,2}$ The first is a series of 164 patients treated in at the Amyloidosis Research and Treatment Center in Pavia, Italy, half of whom received bortezomib, melphalan and dexamethasone (BMDex) - the cases -and the other half, MDex - the controls. ${ }^{1}$ The second study is a series of 138 patients seen at the National Amyloidosis Centre in the UK, half of whom received cyclophosphamide, bortezomib and dexamethasone (CVD) - the cases-and the other half who received cyclophosphamide, thalidomide and dexamethasone (CTD) - the controls. ${ }^{2}$ Both groups of authors used similar and appropriate matching criteria to select their controls (Table 1) with an emphasis on cardiac function, ${ }^{3-5}$ although the Italian patients were matched for more characteristics, including age (Table 1). Despite these efforts by these authors, the question regarding the added value of incorporating bortezomib into first-line therapy among patients with $\mathrm{AL}$ amyloidosis is still not adequately answered. We, therefore, continue to await the results of a randomized clinical trial (NCT01277016).

Patients were treated according to guideline rather than on prospective clinical trial, so there was considerable discretion about dosing level and schedule. In addition, both groups had an algorithm by which the absence of early response prompted a change in regimen in an attempt to improve that response. For the Italian patients, less than a partial response (PR) after two cycles or inadequate response after six cycles prompted a change in therapy. For the UK patients, $<90 \%$ reduction in the difference of serum-free light chain after three cycles prompted a change in therapy. These practices confound the interpretation of depth of best response, particularly for the control arms. Despite this, both bortezomib-containing regimens still yielded significantly higher complete response (CR) rates in both studies: $40-42 \%$ with CVD as compared with $19-25 \%$ with the other two regimens (Table 1). Organ responses were examined in both studies and were disappointingly low throughout, perhaps owing to relatively short follow-up.

Despite the better CR rates, overall survival (OS) and 1-year OS were comparable across the cohorts and the arms at $\sim 60-67 \%$ (Table 1). All OS analyses are limited by their relatively short follow-up, which is 26 months for the Italian cases and controls as well as the UK controls, but only 13 months for the UK cases. It is conceivable that with longer follow-up, differences in OS might emerge.

Further subgroup analyses were performed. One consistent message across both studies was that patients with the highest baseline risk factors fared equally poorly regardless of bortezomib inclusion or exclusion into their upfront regimen. In the UK study, both those cases and the controls who had an NT-proBNP of $\geqslant 8500 \mathrm{ng} / \mathrm{l}$ had a median OS of $\sim 4$ months; those with NTproBNP less than that cut-off enjoyed nearly an 80\% 1-year OS, regardless of the regimen. In the Italian study, there was one subgroup that achieved a significantly better OS when treated with BMDex. Those patients with a New York Heart Association class of $<3$ and an NT-proBNP of $\leqslant 8500 \mathrm{ng} / \mathrm{l}$ who were treated with BMDex had a superior OS compared with their counterpart of MDex-treated patients (2-year OS of $86 \%$ and $\sim 65 \%, P=0.01$, respectively). These OS figures were not corrected for potential confounding factors including the fact that the MDex patients were treated earlier and therefore potentially had fewer salvage options, but taken at face value would suggest that adding bortezomib upfront in lower-risk patients might yield better outcomes. The authors highlighted that this OS difference disappeared when the analysis was limited to those patients who received full dose rather than attenuated dexamethasone.

Both groups performed 6-month landmark analyses to further deconstruct the added value of bortezomib as part of a first-line treatment strategy. Even on landmark analysis, no OS improvement was seen in the UK case match, but there was a slight improvement in OS in the Italian case match.

Progression-free survival (PFS) is not clearly defined in either study. The Italian study reports time to next therapy or death, and finds no significant superiority for the BMDex over the MDex (39 versus 22 months, $P=0.3$ ). In the UK study, the authors report 'PFS,' yet in the Methods, they state that PFS was examined in patients 'achieving a PR or better,' which is the definition for duration of response rather than PFS. The duration of response for the CVD was better than that for the CTD ( 28 versus 14 months, $P=0.04)$. A similar trend was observed after 6-month landmark analysis (not reached versus 19.2 months).

Both groups examined the potential role that salvage bortezomib might play. Fourteen (20\%) of the UK control patients had their therapy switched after three cycles of therapy, 13 of whom received a bortezomib-containing regimen, resulting in upstaging $57 \%$ (8 of 14) of CTD patients to a very good partial response or better. In the Italian study, $40 \%$ of the control patients received second-line therapy. Of those 35 controls, 18 received bortezomibbased therapy and a little more than half of these had a PR or better. When the authors performed a landmark analysis limiting the case group to only those who received bortezomib as salvage, the control group - that is, the upfront bortezomib group-had a longer OS as compared with the sequential MDex followed by bortezomib (median 31 months versus not reached, $P=0.03$ ).

What conclusions can be drawn from these case-controlled studies that we could not glean from early phase $\mathrm{I} / \mathrm{II}$ data ${ }^{6}$ and from other cases series? ${ }^{7,8}$ These studies confirm that high CR rates can be achieved using bortezomib along with an alkylator and corticosteroid, although CR rates are lower than the $65-71 \%$ reported in two other smaller series. ${ }^{7,8}$ The lower $C R$ rate may be explained by the fact that the populations studied in the two case-control studies contained $15 \%$ or fewer Mayo 2004 stage I patients and therefore had high competing rates of death. The second take-home message is the reassurance that patients treated with bortezomib do not appear to have higher early death rates; this is important, as clinical trials using bortezomib have excluded high-risk AL patients., ${ }^{6,9,10}$ The third lesson is the discouraging finding that OS for the highest risk patients with $\mathrm{AL}$ amyloidosis is not improved by adding bortezomib. Prior case series had touted CVD as a potential panacea for high-risk amyloid patients, ${ }^{7,8}$ and it was hoped that a high complete hematologic response rates might result in a higher salvage rate for the most 
Table 1. Comparison of series

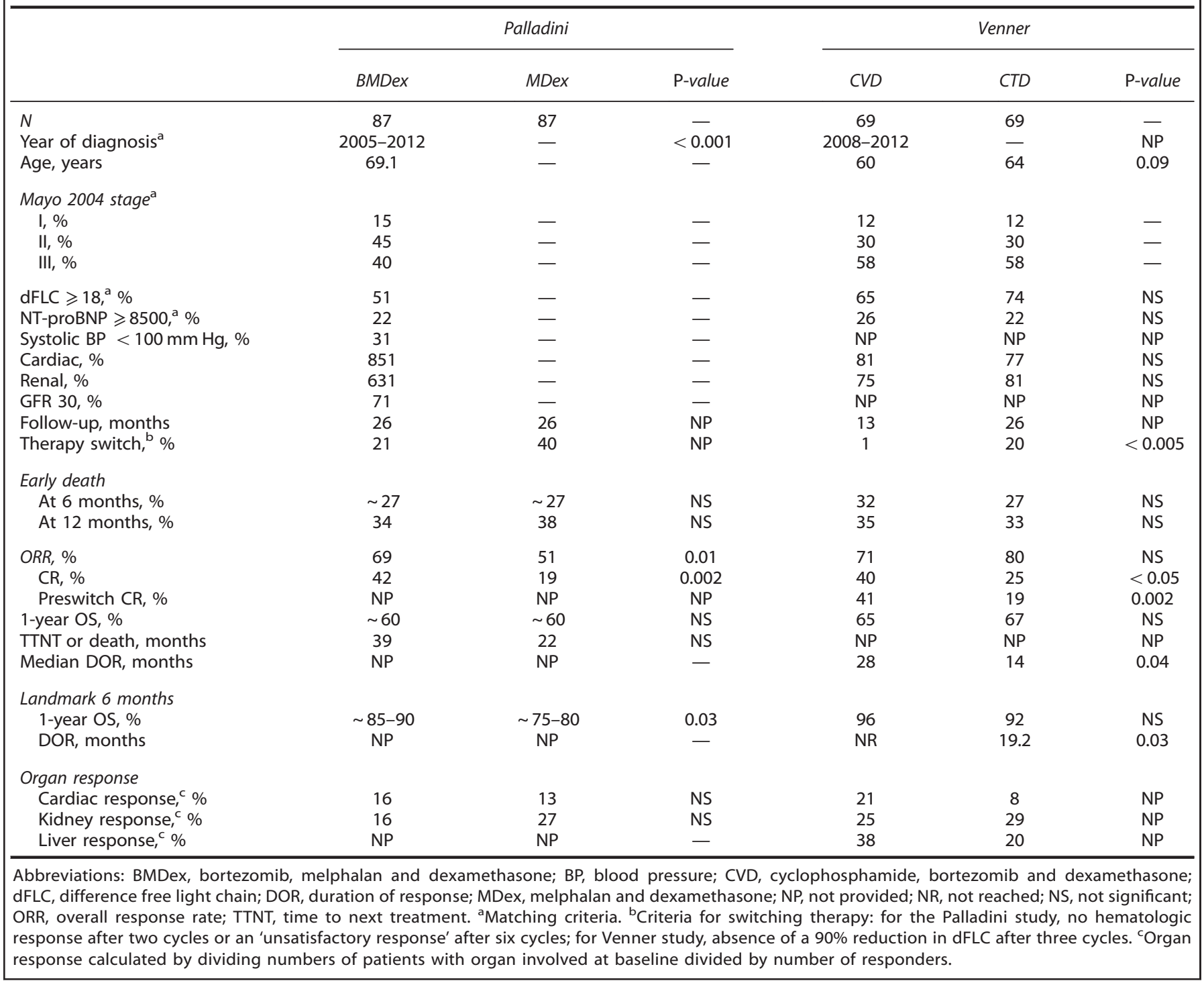

unfortunate patients. The fourth message is that intermediaterisk patients who are not fit enough to receive high-dose dexamethasone are likely to take the greatest advantage from the addition of bortezomib to MDex. The fifth and final message is that there is a suggestion that duration of response may be superior with CVD as compared with CTD.

\section{CONFLICT OF INTEREST}

$A D$ receives research dollars for clinical trials from Millenium, Celgene, Pfizer, and Jannsen.

A Dispenzieri

Division of Hematology, Department of Internal Medicine, Mayo

Clinic, Rochester, MN, USA

E-mail: dispenzieri.angela@mayo.edu

\section{REFERENCES}

1 Palladini G, Milani P, Foli A, Rosin MV, Basset M, Lavatelli F et al. Melphalan and dexamethasone with or without bortezomib in newly-diagnosed $\mathrm{AL}$ amyloidosis: a matched case control study on 174 patients. Leukemia 2014; 28: 2311-2316.

2 Venner CP, Gillmore JD, Sachchithanantham S, Mahmood S, Lane T, Forard D et al. A matched comparison of cyclophophamide, bortezomib and dexamethasone (CVD) versus risk adapted cyclophosphamide, thalidomide, and dexamethasone (CTD) in AL amyloidosis. Leukemia 2014; 28: 2304-2310

3 Dispenzieri A, Gertz MA, Kumar SK, Lacy MQ, Kyle RA, Saenger AK et al. High sensitivity cardiac troponin $\mathrm{T}$ in patients with immunoglobulin light chain amyloidosis. Heart 2014; 100: 383-388.

4 Dispenzieri A, Gertz MA, Kyle RA, Lacy MQ, Burritt MF, Therneau TM et al. Prognostication of survival using cardiac troponins and $\mathrm{N}$-terminal pro-brain natriuretic peptide in patients with primary systemic amyloidosis undergoing peripheral blood stem cell transplantation. Blood 2004; 104 1881-1887.

5 Wechalekar AD, Schonland SO, Kastritis E, Gillmore JD, Dimopoulos MA, Lane $\mathrm{T}$ et al. A European collaborative study of treatment outcomes in 346 patients with cardiac stage III AL amyloidosis. Blood 2013; 121: 3420-3427.

6 Gasparetto C, Sanchorawala V, Snyder RM, Matous J, Terebelo HR, Janakiraman N et al. Use of melphalan (M)/dexamethasone (D)/bortezomib in AL amyloidosis. J Clin Oncol 2010; 28(15 Suppl): 8024.

7 Mikhael JR, Schuster SR, Jimenez-Zepeda VH, Bello N, Spong J, Reeder CB et al. Cyclophosphamide-bortezomib-dexamethasone (CyBorD) produces rapid and 
complete hematologic response in patients with $\mathrm{AL}$ amyloidosis. Blood 2012; 119: 4391-4394.

8 Venner CP, Lane T, Foard D, Rannigan L, Gibbs SD, Pinney JH et al. Cyclophosphamide, bortezomib, and dexamethasone therapy in AL amyloidosis is associated with high clonal response rates and prolonged progression-free survival. Blood 2012; 119: 4387-4390.
9 Reece DE, Hegenbart U, Sanchorawala V, Merlini G, Palladini G, Blade J et al. Efficacy and safety of once-weekly and twice-weekly bortezomib in patients with relapsed systemic AL amyloidosis: results of a phase $1 / 2$ study. Blood 2011; 118: 865-873.

10 Reece DE, Sanchorawala V, Hegenbart U, Merlini G, Palladini G, Fermand JP et al. Weekly and twice-weekly bortezomib in patients with systemic AL amyloidosis: results of a phase 1 dose-escalation study. Blood 2009; 114: 1489-1497. 